\title{
A Brief Review of Recent Developments in the Integration of Deep Learning with GIS
}

\begin{abstract}
The interaction of Deep Learning (DL) methods with Geographical Information System (GIS) provides the opportunity to obtain new insights into environmental processes through the spatial, temporal and spectral resolutions as well as data integration. The two technologies may be connected to form a dynamic system that is incredibly well adapted to the evaluation of environmental conditions through the interrelationships of texture, size, pattern, and process. This perspective has acquired popularity in multiple disciplines. GIS is significantly dependant on processors, particularly for 3D calculations, map rendering, and route calculation whereas DL can process huge amounts of data. DL has received a lot of attention recently as a technology with a plethora of promising results. Furthermore, the growing use of DL methods in a variety of disciplines, including GIS, is evident. This study tries to provide a brief overview of the use of DL methods in GIS. This paper introduces the essential DL concepts relevant to GIS, the majority of which have been published in recent years. This research explores remote sensing applications and technologies in areas such as mapping, hydrological modelling, disaster management, and transportation route planning. Finally, conclusions on contemporary framework methodologies and suggestions for further studies are provided.
\end{abstract}

Keywords: deep learning, GIS, integration, classification, remote sensing

Received: 13 September 2021; accepted: 22 December 2021

(C) 2022 Authors. This is an open access publication, which can be used, distributed and reproduced in any medium according to the Creative Commons CC-BY 4.0 License.

1 Research Scholar, Centre for Water Resources, Institute of Science and Technology, Jawaharlal Nehru Technological University Hyderabad, Kukatpally, Hyderabad, India, email: shyama7789@gmail.com, (1) https://orcid.org/0000-0002-7935-448X

2 Centre for Water Resources, Institute of Science and Technology, Jawaharlal Nehru Technological University Hyderabad, Kukatpally, Hyderabad, India, email: mvssgiridhar@gmail.com, (i) https://orcid.org/0000-0001-9029-9621 


\section{Introduction}

With the availability of a large number of spatial resources and data, processing and management are becoming increasingly common. These spatial data provide crucial geographical information. As a result, in order to process this information, best practices and applicable methodologies must be devised. The data can reveal comprehensive details of events or locations created through modelling and analysis. This opens up numerous chances for improving government services in terms of best governmental segmentation, consumer interaction, and process optimization. Artificial intelligence, such as deep learning techniques, is one such technique that can evaluate and manage such massive amounts of data. DL has advanced rapidly in recent years, outperforming humans in recognition, detection, translation, and so on. DL has yielded a plethora of intriguing tools in a variety of domains, particularly GIS (Geographical Information System). The union of deep learning and geographic information systems (GIS) creates enormous prospects.

GIS discipline enables users to comprehend spatial dimensions and relate them to actual geographical properties or phenomena. GIS is a large user-friendly system that enables quick data storage and access. GIS can be utilized for a variety of reasons, including disaster management and monitoring, watershed management, demographic research, urban sprawl assessment, risk evaluation, food productivity, irrigation supply and management, and so on. GIS provides for the storage of large amounts of data in a database that can be accessed at any time and from any location.

Multidisciplinary fields, such as environmental processes with constant change and linkages, necessitate the development of novel approaches and solutions. There have been various overviews of deep learning in multidisciplinary techniques, such as uses of DL in remote sensing [1]. Zhang et al. [2] present a detailed overview and general framework of DL for RS data on image processing, pixel-based classification, pan-sharpening, spectral feature classification, and target recognition. The majority of the review papers on deep learning in remote sensing cover image pre-processing areas such as pan-sharpening, image fusion, registration, scene classification and object recognition, segmentation, and object-based classification [2-4]. Ball et al. [5] conducted a complete evaluation on deep learning on remote sensing photos, with substantial remote sensing studies where deep learning can be integrated. Review on areas such as classification, 3D analysis, traffic sign recognition, depth estimation, object identification and tracking, weather forecasting, object detection, and image enhancement have been briefly discussed along with unsolved challenges and opportunities for DL in RS.

In other words, applications of DL in the field of remote sensing have been reviewed, although DL combined with the integration of GIS utilizing remote sensing data has been less explored. There is a high degree of necessity for the RS and GIS communities to use DL techniques in various fields of GIS, therefore a review is required to understand the DL and GIS integration. The study primarily discusses 
the applications of the integration of DL with GIS. The paper is organized into the following sections: DL neural networks, the integration of GIS and DL, applications of the integration of DL with GIS, gaps and future trends, discussion and conclusion.

\section{Deep Learning Neural Networks}

DL is a branch of machine learning dealing with algorithms influenced by the structural system and operation of the brain termed artificial neural networks. Since the 1980s, neural networks have been employed to classify remote sensing data [6]. A substantial number of studies have gradually been building proving their capabilities, e.g., [7-14]. DL solves problems and makes predictions using computergenerated neural networks that are intrigued by and resemble the human brain. A DL structure comprises vast amounts of data, sophisticated computational resources such as algorithms, graphics processing units (GPUs), and network architects. Training (or learning) and interpretation (or prediction) are the two stages of neural networks that allude to development vs production. The number of layers and kind of neural network is chosen by the developer, while the weights are determined through training. There are several types of DL algorithms available today, with the most widely used being discussed in the sections below.

\subsection{Autoencoders}

Autoencoders are neural networks made up of input, hidden, and output layers. An autoencoder can learn different coding patterns. In an autoencoder, the numbers of nodes are the same in both the output and input layers. The autoencoder must identify its inputs rather than target values based on the output vector.

\subsection{Multi-layer Perceptrons (MLP)}

MLP is artificial neural network that is feedforward. The multilayer perceptron is one of the most fundamental DL models, consisting of a sequence of fully connected layers. Each successive layer is a collection of nonlinear functions based on the weighted sum of all outputs from the previous one. These networks are widely utilized in voice detection and other machine learning applications.

\subsection{Convolutional Neural Networks (CNN)}

A convolutional neural network (CNN) is a feedforward multilayer perceptron model. The initial layers of a deep network identify characteristics, while subsequent layers reassemble these characteristics into higher-level input parameters. CNN is excellent in visual recognition and identifying various visual patterns. Aside from image processing, CNN has been successfully applied to video identification and a variety of natural language processing tasks. 


\subsection{Recurrent Neural Networks (RNN)}

The convolutional model takes a given quantity of inputs and outputs a fixedsized vector after a predetermined number of steps. Recurrent networks enable us to act on vector segments in both input and output. A recurrent network, unlike a typical neural network, may include connections that loop back into previous layers (or into the same layer). This feedback enables RNNs to retain recollection of previous inputs and simulate issues over time. Long Short-term Memory is a widely known RNN. This type of network is utilized in the creation of chatbots and text-to-speech systems.

\subsection{Deep Reinforcement Learning to Neural Networks}

This adopts a very different technique. It places an entity in a setting with specific boundaries, defining productive and undesirable action and an overarching goal to achieve. In some aspects it is comparable to supervised learning; within this, programmers must provide algorithms with well-defined goals and specify positive and negative reinforcement. The learning algorithm modifies the entity's action during training. The learning algorithm's objective is to identify the best action that maximizes the long-term reward obtained throughout the activity. Deep reinforcement learning can be utilized in planning and control applications.

\section{The Integration of GIS and DL}

Massive increases in computing power are rapidly providing new possibilities, particularly in DL, and GIS is strongly reliant on such computational capacities. Integration of GIS with DL has proven to be a potential utility, particularly in 3D modelling, map generation, and route calculation. When remotely sensed data are integrated with other geographical variables structured inside a GIS framework, the analytical capability of the system is increased as a result of the integration. GIS is helpful in every workflow of AI. For example, photographs recognized using DL may be geo-tagged in GIS, and images can be detected in GIS using an algorithm. ANN requires a data storage, retrieval, analysis, and visualization environment that is compatible. This may be provided by integrating ANN with GIS [15].

The integration of GIS with DL will be helpful in various applications such as the classification of RS images or attribute data analysis. Combining a GIS database with powerful DL models will aid in enhanced environmental mapping and object search/detection in integrated database [16]. Because neural network classifiers operate as generic pattern recognition systems and, furthermore, because they do not require a previous statistical model for their input data, they are very versatile when it comes to combining different types of data. As a result, they may be utilized to include GIS data into remote sensing image classification [17]. Several experiments 
involving the usage of GIS data have previously been published. For example, Benediktsson et al. [9] used topographic data in conjunction with Landsat Multi-spectral Scanner (MSS) data to map ground cover. Another experiment (Joint Research Committee 1991) employed landscape height from a digital terrain model (DTM) as an additional input to a multi-layer perceptron neural network with two dates of SPOT HRV images. The experiment's goal was to categorize land cover in satellite images. In this example, including DTM information from a GIS boosted overall classification accuracy while also cutting training time for the neural network in half. So far, the use of GIS data in neural networks is a relatively recent topic, with minimal experimentation. However, the inadequate data available suggests that this would be a profitable strategy to pursue [18].

\section{Applications of Integration of DL with GIS}

Remote sensing provides a continuous and consistent stream of information about the Earth, and geographic information systems (GIS) are a methodology for dealing with all of this geographic data. Remote sensing combined with geographic information systems (GIS) aids in decision-making for a variety of applications, including sustainable natural resource management, network design of protected areas, and tackling global change issues. GIS is capable of collecting and processing real time data. This progression aided research and mapping systems, but real-time geospatial data can also help consumers. Real-time geospatial intelligence can also be used to track the progress of natural disasters. The analysis of anything from climate variability to social media posts offers suggestions in desperate need of rescue operations.

GIS permits us to work with raster and vector data, which can be readily transformed and worked with. Using existing technologies and operational systems, integrating satellite, airborne, and terrestrial RS systems is possible to generate a scale-dependent set of observations [19]. It must be easy to produce image statistics within polygons and then directly return that information to the GIS database as polygon attributes [20]. They are also investigating the possibility of using RS and GIS to display and enhance the presentation of statistical data and identify and present numerous opportunities to the GIS research community [21]. GIS has evolved into a must-have tool for processing, analyzing, and visualizing spatial data. Geographic data and geographic information systems (GIS) are so crucial in environmental disciplines that we now consider them essential components of research, education, and policy [22]. There are numerous software programs available to aid in GIS decision making. GIS software is tailored for specific sorts of decision making. As a result, the software is available based on the needs of the user. These software systems respond effectively to an individual input, and they may be suitable to one or more broad domain applications, but they are also restrictive. Large 
and complicated data sets with many features might cause geoprocessing tools to perform poorly or fail. As a result, running a sophisticated model in GIS may be unfeasible, resulting in output errors. This is where DL techniques come into play, as they can run algorithms with high resolution and complicated datasets while providing high accuracy results.

The Scopus, Google Scholar, and Web of Science databases were used to review publications that included the integration of DL with GIS. Peer-reviewed publications were evaluated and categorized based on their field of application. The findings demonstrated that DL might be used in virtually any GIS application. Each theme was identified, and the review conducted focusing primarily on the topics provided below to emphasize DL's significance and prospective development in the field of GIS.

\subsection{Classification/Mapping}

The classification of remote sensing images is the most commonly performed application. Image classification is a methodology of defining pixels. Depending on which aspects of the images can be detected and categorized, the images have varying spectral and spatial resolutions. GIS has executed image classification for a considerable time, and DL has also been used to perform image classifications. As a tool for replicating the human brain's intuitive reasoning process, ANN can map complicated systems with no prior understanding of them. The handling of geographic information has been made possible by the development of a system that combines ANN with a GIS. Yang and Rosenbaum [15] effectively connected ANN to GIS with the NRSE algorithm, resulting in a tool to provide decision support for technical challenges and harbor management. Sediment prediction inside Gothenburg harbor has been used to illustrate its utility. Because natural systems are generally complex, establishing a deterministic model is challenging, yet quantifiable representation is needed. DL and GIS integration were also employed in agriculture.

Mansourian et al. [23] created zonation maps in Kurdistan province, Iran, using GIS-based LR models to forecast the dominant weed presence in dryland chickpea and winter wheat fields. With high MSE values, ANN may create the best-suited models for predicting all prevalent weeds. Xiong et al. [24] mapped mineral prospectivity through big data analytics and DL. The results show that the mapped prospectivity regions have a significant spatial association with known mineralization sites and show that big data analytics assisted by DL methods is a promising approach to explore for application in mineral prospectivity mapping. Demertzis et al. [25] developed a novel and highly successful geographic object-based scene classification system in remote sensing images, based on an innovative ResidualNeural Network (ResNet) architecture. This network also implements a Large-Scale Geospatial Data Analysis method that uses ResNet to manage latency, throughput, and 
fault-tolerance while concurrently utilizing learning processes as optimally and efficiently as feasible. Zhao et al. [26] prepared land use characteristics of Bay Area using GIS and DL. Four indices of land use characteristics for 122 zones were extracted from GIS and merged with planning information using DNN technique. Based on the study error distribution, the results suggest that the proposed technique has good accuracy and looks to be promising and resilient. The total inaccuracy is rather modest. Wu and Biljecki [27] search for sustainable roofing in cities. This research was an inspiration from a greenery counterpart Treepedia. An automated methodology that combines DL with geospatial technology for detecting sustainable rooftops in cities. The project successfully detected sustainable roofs with an accuracy of $100 \%$. The models are precise enough to give meaningful city-wide spatial and quantitative evaluations, both in terms of the placement and amount of sustainable rooftop devices. Several research combining DL and GIS indicate that the need for precise and effective scene classification can significantly contribute to the creation of new types of decision support systems [25].

\subsection{Traffic and Route Planning}

Transport infrastructure is inherently crucial to national development since it is a need in life and has an impact on all elements of human existence [28]. Moving from one location to another in any metropolis across the world is an endurance test; regardless of money or social standing, the conditions under which people commute are getting increasingly challenging. GIS for road and highway safety allows for the collection and analysis of reliable vehicle accident data. Historical records aid in understanding where incidents have happened in the past and what precautions may be taken to avoid them in the future.

Campbell et al. [29] detected and mapped traffic signs from Google Street View photos using DL and GIS to create an autonomous system for identifying traffic signs to aid in the monitoring and maintenance of traffic assets. Experiments conducted on the study area's road network yielded a detection accuracy of $95.63 \%$ and classification accuracy of $97.82 \%$. The suggested automated approach to the detection and localization of street sign technology has shown great potential for application by local government agencies. Mubin et al. [30] detected oil palm by integrating a GIS with a DL strategy. This study aims to close the gap by using two distinct convolution neural networks (CNNs) to independently recognize juvenile and mature oil palm, along with GIS throughout the data processing and result. Li et al. [31] proposed a U-Net-Based semantic segmentation method to extract building footprints. Multiple public databases of GIS maps of the four cities were integrated with high resolution satellite imagery. The method extracted the building footprint from multi-spectral resolution images using SpaceNet dataset. The proposed technique yields a total F1-score of 0.704 , a $1.1 \%$ improvement to $12.5 \%$. Kearney et al. [32] utilized RapidEye, participative GIS, and DL to extract and update the unpaved roads. 
This study created and tested a technique for automatically extracting unpaved road pixels using a convolutional neural network over a vast forested region in Western Canada $(\mathrm{CNN})$. The $\mathrm{CNN}$ results were then used to update an existing government road network to see whether and how it would alter. Images and a ground-truth dataset were acquired using smartphones by enterprises that are currently active and driving in the region. Using participative techniques to acquire ground data proved advantageous in gathering training and validation data across a wide region at a reasonable cost. The performance of the road extraction was equivalent to that of others who used extremely high-resolution images; recall accuracy was 89-97\% and precision was $85-91 \%$. Servizi et al. [33] used geospatial context and artificial neural networks to detect stops in smartphone-based trip surveys. To address these uncertainties, geographical time-series, such as GPS, were coupled with spatial context information collected from Open Street Map, resulting in multi-dimension tensors. This multidimensional representation of time-series fusion with geographical context and the appropriate specialized ANN classifier makes the primary contribution. The results reveal a 3 to $6.5 \%$ improvement in stop detection scores over the baselines.

Accurate and up-to-date road network data is critical for land management and disaster response, unpaved road especially in the villages and wooded areas are not easy to generate using traditional techniques [32]. Malaainine et al. [34] created a DL model with GIS-based vehicle tracking algorithms. The spatio-temporal GIS (Geographic Information System) trails of moving vehicles were generated using a Convolutional Neural Network (CNN) that processed high-resolution satellite imagery. Bi et al. [35] created a sustainable urban road management by GIS and neural network. The integrated model determines routes, time and course of action for travelers in an urban network. Compared to realistic trip data, the bi-level optimization method may cut the average journey time by roughly $20 \%$ and energy usage by $10 \%$.

\subsection{Hydrological Models}

Geographical distributions on the earth surface are intricate and complex. Modelling these distributions as a replica require a number of variables and data and are time dependent. The spatial representation of these distributions is critical, but GIS and DL can assess and predict this complex problem at high accuracy. Yang and Rosenbaum [36], provided a brief overview on DL NN with an emphasis on the GIS overlay mechanism. This integrated approach uses ANN within GIS system for the application of mapping. The method was used to forecast sediment in the harbor of Gothenburg in this study. Dixon [37] developed groundwater vulnerability model using the neuro-fuzzy technique and GIS. The main goal of this study is to investigate the sensitivity of neuro-fuzzy models used to forecast groundwater vulnerability in a geographical context. The neuro-fuzzy models, on the other hand, 
should only be utilized as part of a larger assessment of groundwater vulnerability that includes GIS, remote sensing, and solute transport modelling, as well as functional, mechanistic, and stochastic models.

Almasri and Kaluarachchi [38] predicted the nitrate load in groundwater through modular neural networks (MNN). The field data of nitrogen loading and recharge data were prepared from GIS tools and forecasting was modelled in MNN. In the preparation and processing of the MNN input-output data, the methodology relies on geographic information system (GIS) tools. MNN's long-term models, which were used to evaluate the efficacy of future management options, correctly identified the locations with relatively higher nitrate concentrations. Rohmat et al. [39] developed a model called River GeoDSS which is a decision support system that includes database management, and graphical user interfaces. It is fully integrated in a geographic information system (GIS) for geospatial modeling and analysis. The trained ANN was then re-inserted into River GeoDSS and used to simulate river basin management. All DNN model development takes place entirely within River GeoDSS in the updated version. Apaydin and Sattari [40], integrated GIS and DL for precipitation modeling dependent on spatiotemporal variables. To obtain precipitation, the hybrid model was applied to Turkey's coastline. The study utilized DL to estimate the amount of precipitation, and a distribution map was created using a DL-GIS hybrid.

Patault et al. [41] applied a deep neural network and a GIS model to predict sediment discharge under various land scenarios. The model produced a standard deviation that was exactly in line with the GEV distribution. The model also serves as a decision-making tool for providers of drinking water and land use planners. Urban flood modelling utilizing deep-learning techniques was developed by Lei et al. [42]. Flood hazard mapping was produced using a deep convolution neural network model and a GIS. The flood inventory of 295 flooded sites was provided by the GIS database, which was used as the response variable, and predictor variables were flood affecting factors in DL. Hydrological models such as flood modelling can be quick and reliable if a large inventory is available.

\subsection{Disaster Management}

Natural and man-made calamities threaten several regions of the world, including India. GIS, RS, and DL are useful in disaster management and decision-making scenarios. Disaster maps can be created to depict risk and impact zones. As a result, GIS and DL are beneficial for mapping considering all the parameters influencing the calamities, which may aid in a better understanding of site risk and planning. Stassopoulou et al. [43] demonstrate the technique's validity by determining the parameters to be utilized in a Bayesian network built to incorporate GIS data to estimate the probability of the desertification of burnt forest areas in the Mediterranean region. Choi et al. [44] combined ANN and GIS to create landslide susceptibility 
maps. DEM, NDVI, and land cover were derived from ASTER images in the GIS. Using the geographical database that was created, the correlations between the detected landslide locations and six associated variables were detected and quantified using frequency ratio (FR), logistic regression (LR), and artificial neural network (ANN) models. Maps obtained from the integration of GIS and ANN showed higher accuracy than the individual landslide maps.

Hamdi et al. [45] employed GIS and DL on high-resolution data to estimate forest degradation. The study used CNN in GIS software with an algorithm for detecting and mapping forest damage. The results were accurate to $92 \%$. Given the difficulty of locating regions of fallen trees, these results are significant. The study demonstrated the quick efficiency of GIS and tremendous potential of DL on high resolution maps for post disaster damage assessment. Bui et al. [46] used a DL Neural Network with a GIS dataset to forecast the vulnerability to flash floods. The flash flood inventory data is used to calculate the pixelwise flash flood susceptibility for the study region employing DLNN. As a result, the suggested hybridization of GIS and DLNN may well be a viable alternative for assisting government authorities and engaging parties in the development of efficient flash flood mitigation methods and land-use planning. Radke et al. [47] created the FireCast program, which combined DL and GIS techniques. The FireCast predicts the wildfire will spread throughout the area. Based on previous fire data and with minimal computing resources, FireCast can accurately forecast whether regions surrounding a blazing wildfire are at high risk of future wildfire spread. Nhu et al. [48] developed algorithms to predict the likelihood of landslides in a tropical environment. In GIS, landslide affecting variables and polygons were created, utilizing a deep neural network to map all feasible regions. The algorithm divides the area into two distinct classes: landslide and non-landslide.

Wu et al. [49] assess the depth of urban floods using DL and GIS. The rainfall return period was utilized to produce the flood condition map in GIS, and the flood depth was predicted using a regression model in DL. The findings of this study can serve as a firm justification for urban flood management and drainage. Bragagnolo et al. [50] created the r.landslide tool to map the susceptibility of landslides. The application makes use of open source GIS tools as well as ANN. Python is used as a built-in program with Artificial Neural Network (ANN) fed environmental factors and landslide databases. r.landslide can more precisely detect and specify vulnerable locations. With natural disasters growing more frequent and severe, disaster risk mitigation is becoming increasingly important. GIS and DL are extremely useful in mitigation and preparation measures. Real-time geographic data can help optimize the allocation of response resources. It also serves as a decisionmaking tool in disaster management. Global governments and businesses should collaborate to develop novel tools and methodologies for developing an effective disaster management strategy that takes use of the integration of DL and GIS approaches. 


\subsection{Urban Planning}

Urban planning entails the design of cities as well as the creation of plans for future development and expansion. GIS and DL can give vital information and enhanced strategic decision making with a single source for current and historical data and maps. Ma and Cheng [51] calculated building energy incorporating GIS and big data. This study presents a framework for calculating the building EUI at the urban scale using a geographic information system integrated data mining approach, including pre-processing, feature selection, and algorithm optimization. Li et al. [31] developed a GIS and DL model to estimate the value of urban land. The urban land price is identified by taking into consideration variables affecting urban land price and spatial features and integrating them in DL. The linear relationship and causal link of influencing variables and land price are automatically detected by creating the deep hybrid neural network with spatial characteristics.

Re Cecconi et al. [52] used GIS and ANN to assess the possibilities for energy retrofits in school buildings. The potential for energy efficiency was assessed using eight Artificial Neural Networks. Finally, data were geolocated and further analyzed to aid in the development of energy retrofit strategies for the most essential regional locations. The case study results shown that energy retrofit strategies for school buildings are mostly supported and developed utilizing open data, neural networks, and GIS. Kucklick et al. [53] combined GIS and CNN to quantify the influence of location data on property value in Philadelphia. The study discovered that significant geographical data and high quality images were necessary to improve the model's accuracy. Using two datatypes is challenging and interesting, but it provides improved resilience against severe anomalies. Zhang et al. [54] identify and depict various urban scenarios using DL and massive street-view datasets. The landsense indices were used to quantify the relationship between public perception and urban scenes. The model's outcome demonstrated a good performance in natural landscapes in Beijing's built-up areas and a negative psychological impact in industrial areas. This unique technique has the potential to aid in urban planning and management from the perspective of residents. Pepe et al. [55] employed DL in a GIS framework to create a 3D city model from multispectral satellite images. Different techniques in geomatics software were used to extract building elevations from DEM for building polygons of varying positions. Using an algorithm in ArcGIS Pro, a method of pan sharpening, followed by a digital surface model, and lastly the extraction of building height was devised. The model quickly generated 3D buildings as prismatic solids with flat roofs. This unique strategy is yet another technique that assists stakeholders and administrations in the urban planning and design domain.

GIS and DL provide urban planners with more data visibility. They track changes over time, assess the viability of proposed initiatives, and forecast their environmental impact. 


\section{Gaps and Future Trends}

DL approaches can generate classifications or predictions about a particular target based on a collection of input attributes, and GIS can produce the necessary geographical input variables for such an DL model [47]. However, there are constraints when they are performed independently. This may be avoided by combining GIS with DL. Big data technology allows for the integration of many predictors and variables, which is not feasible with GIS. To carry out such modelling, however, DL requires a huge spatial database, which may be collected via a GIS database. DL and GIS can explore difficult tasks with complex and dynamic data. Most geographical characteristics are dynamic, necessitating complicated modelling, which may be accomplished using neural networks and other spatial data from the GIS database. This will enhance the accuracy of the modelling. Further study on similar issues is necessary.

Environmental degradation indicators, environmental monitoring, climate change, hydrological models such as groundwater and surface water modelling, assessment of soil erosion, soil moisture, urban sprawl analysis, water distribution network analysis, disaster risk analysis, site suitability, coral reefs, and sustainability have all been studied using remote sensing and GIS. However, with a high-resolution database and extensive modelling, the findings may be inaccurate. By combining GIS with deep neural networks, these applications may be studied further and achieve high-accuracy results. Future applications of DL with GIS include susceptibility mapping of various natural hazards and investigating more complex feature selection or dimension reduction methods to improve the prediction performance of DL methods. Future studies may incorporate historical data from various regions and explore different resolution images, input variables, and scenario analysis. Integrating DL with GIS aids in the development of a better decision-making tool. The approach may be utilized as a support tool for the rapid and efficient generation of various maps by organizations involved in disaster management, water resources, the environment, and land use planning [50].

\section{Discussions and Conclusions}

DL is a fast-developing discipline that enables data scientists to use cutting-edge research while utilizing an industrial-strength GIS. Because of this appealing characteristic, neural networks are increasingly being used to model complicated physical processes, and a lack of precise field data predominate [38]. GIS technologies can assist with every phase of the data science workflow, including data preparation and exploratory data analysis, training the model, executing spatial analysis, and lastly, distributing results via online layers and maps and driving field activities. DL algorithms, on the other hand, can handle tasks with complex data structures and modelling, yielding high accuracy with greater flexibility and generalization capability. 
GIS provides a well-established platform for spatial data analysis, to which ANN can offer a powerful mechanism for decision support. Because the findings can be accessible by mobile devices in the field for high-accuracy ground-truthing and different mapping that can be synced back into the database, the integration with the ArcGIS Platform provides for a simplified workflow [45]. As a result, it is possible to infer that the suggested GIS-DL hybridization can be a promising tool for assisting government authorities and engaging stakeholders in flash flood prevention and land-use planning [46].

This paper investigated multiple research areas where DL and GIS have been integrated. A total of 55 papers from the Scopus, Web of Science, and Google Scholar databases were reviewed. The articles were divided into the following categories, namely classification and mapping, traffic and route planning, hydrological models, disaster management, and urban planning. The brief review of these research papers demonstrates the significance of combining DL and GIS for analyzing various continuous and unstable data. The focus of the paper was on five major applications. A detailed and comprehensive assessment of the approach used with the DL algorithm, as well as its performance analysis, can be undertaken. Further investigations on real-time geospatial intelligence employing DL and GIS to analyze the progression of natural disasters, climate variability, and real-time rescue operations can be performed.

\section{Author Contribution}

Author 1: conceptualization, methodology, investigation, resources, data curation, writing - original draft preparation, writing - review and editing.

Author 2: conceptualization, review, editing, supervision.

\section{References}

[1] Liu Y., Chen X., Wang Z., Wang Z.J., Ward R.K., Wang X.: Deep learning for pixel-level image fusion: Recent advances and future prospects. Inf. Fusion, vol. 42, 2018, pp. 158-173. https://doi.org/10.1016/j.inffus.2017.10.007.

[2] Zhang L., Zhang L., Du B.: Deep learning for Remote Sensing Data: A Technical Tutorial on the State of the Art. IEEE Geoscience and Remote Sensing Magazine, vol. 4, no. 2, 2016, pp. 22-40. https://doi.org/10.1109/MGRS.2016.2540798.

[3] Ma L., Liu Y., Zhang X., Ye Y., Yin G., Johnson B.: Deep learning in remote sensing applications: A meta-analysis and review. ISPRS Journal of Photogrammetry and Remote Sensing, vol. 152, 2019, pp. 166-177. https://doi.org/10.1016/ j.isprsjprs.2019.04.015.

[4] Zhu X.X., Tuia D., Mou L., Xia G., Zhang L., Xu F., Fraundorfer F.: Deep Learning in Remote Sensing: A Comprehensive Review and List of Resources. IEEE Geoscience and Remote Sensing Magazine, vol. 5, no. 4, 2017, pp. 8-36. https://doi.org/10.1109/MGRS.2017.2762307. 
[5] Ball J.E., Anderson D.T., Chan C.S.: Comprehensive survey of deep learning in remote sensing: theories, tools, and challenges for the community. Journal of Applied Remote Sensing, vol. 11(4), 2017, 042609. https://doi.org/10.1117/ 1.JRS.11.042609.

[6] Abdi A.M.: Land cover and land use classification performance of machine learning algorithms in a boreal landscape using Sentinel-2 data. GIScience \& Remote Sensing, vol. 57(1), 2020, pp. 1-20. https://doi.org/10.1080/15481603.2019.1650447.

[7] Key J., Maslanik J.A., Schweiger A.J.: Classification of Merged AVHRR and SMMR Arctic Data with Neural Networks. Photogrammetric Engineering \& Remote Sensing, vol. 55(9), 1989, pp. 1331-1338.

[8] Hepner G.F., Logan T., Ritter N., Bryant N.: Artificial neural network classification using a minimal training set: comparison to conventional supervised classification. Photogrammetric Engineering \& Remote Sensing, vol. 56, no. 4, 1990, pp. 469-473.

[9] Benediktsson I.A., Swain P.H., Ersoy O.K.: Neural network approaches versus statistical methods in classification of multisource remote sensing data. IEEE Transactions on Geoscience and Remote Sensing, vol. 28, 1990, pp. 540-552. https:// doi.org/10.1109/TGRS.1990.572944.

[10] Bischof H., Schneider W., Pinz A.J.: Multispectral classification of Landsat-images using neural networks. IEEE Transactions on Geoscience and Remote Sensing, vol. 30, no. 3, 1992, pp. 482-490. https://doi.org/10.1109/36.142926.

[11] Kanellopoulos I., Varfis A., Wilkinson G.G., Mégier J.: Land-cover discrimination in SPOT HRV imagery using an artificial neural network - a 20-class experiment. International Journal of Remote Sensing, vol. 13, no. 5, 1992, pp. 917-924. https://doi.org/10.1080/01431169208904164.

[12] Downey I.D., Power C.H., Kanellopoulos I., Wilkinson G.G.: A performance comparison of Landsat Thematic mapper land cover classification based on neural network techniques and traditional maximum likelihood algorithms and minimum distance algorithms. [in:] Proceeding of the Annual Conference of the Remote Sensing Society, The Remote Sensing Society, Nottingham 1992, pp. 518-528.

[13] Civco D.L.: Artificial Neural Networks for Land Cover Classification and Mapping. International Journal of Geographical Information Systems, vol. 7, 1993, pp. 173-186. https://doi.org/10.1080/02693799308901949.

[14] Yoshida T., Omatu S.: Neural network approach to land cover mapping. IEEE Transactions on Geoscience and Remote Sensing, vol. 32, no. 5, 1994, pp. 1103-1109. https://doi.org/10.1109/36.312899.

[15] Yang Y., Rosenbaum M.S.: Artificial neural networks linked to GIS for determining sedimentology in harbours. Journal of Petroleum Science and Engineering, vol. 29(3-4), pp. 213-220. https://doi.org/10.1016/S0920-4105(01)00091-2.

[16] Weng Q.: Remote Sensing and GIS Integration. McGraw-Hill Professional Publishing, 2009. 
[17] Wilkinson C.R., Chou L.M., Gomez E., Ridzwan A.R., Soekarno S., Sudara S.: Status of coral reefs in Southeast Asia: threats and responses. [in:] Ginsburg R.N. (ed.), Proceedings of the Colloquium on Global Aspects of Coral Reefs: Health, Hazards and History, June 10, 11, 1993, Rosenstiel School of Marine \& Atmospheric Science, University of Miami, Miami 1994, pp. 311-317.

[18] Wilkinson G.G.: A review of current issues in the integration of GIS and remote sensing data. International Journal of Geographical Information Systems, vol. 10(1), 1996, pp. 85-101. https://doi.org/10.1080/02693799608902068.

[19] Walsh S.J., Butler D.R., Malanson G.P.: An overview of scale, pattern, process relationships in geomorphology: a remote sensing and GIS perspective. Geomorphology, vol. 21(3-4), 1998, pp. 183-205. https://doi.org/10.1016/S0169-555X(97)00057-3.

[20] Hinton J.C.: GIS and remote sensing integration for environmental applications. International Journal of Geographical Information Systems, vol. 10(7), 1996, pp. 877-890. https://doi.org/10.1080/02693799608902114.

[21] Bhunia G.S., Dikhit M.R., Kesari S., Sahoo G.C., Das P.: Role of remote sensing, geographical information system (GIS) and bioinformatics in kala-azar epidemiology. Journal of Biomedical Research, vol. 25(6), 2011, pp. 373-384. https://doi. org/10.1016/S1674-8301(11)60050-X.

[22] Goodchild M.F.: Geographic Information Science and Systems for Environmental Management. Annual Review of Environment and Resources, vol. 28(1), 2003, pp. 493-519. https://doi.org/10.1146/annurev.energy.28.050302.105521.

[23] Mansourian S., Darbandi E.I., Mohassel M.H.R., Rastgoo M., Kanouni H.: Comparison of artificial neural networks and logistic regression as potential methods for predicting weed populations on dryland chickpea and winter wheat fields of Kurdistan province, Iran. Crop Protection, vol. 93, 2017, pp. 43-51. https://doi. org/10.1016/j.cropro.2016.11.015.

[24] Xiong Y., Zuo R., Carranza E.J.M.: Mapping mineral prospectivity through big data analytics and a deep learning algorithm. Ore Geology Reviews, vol. 102, 2018, pp. 811-817. https://doi.org/10.1016/j.oregeorev.2018.10.006.

[25] Demertzis K., Iliadis L., Pimenidis E.: Large-Scale Geospatial Data Analysis: Geographic Object-Based Scene Classification in Remote Sensing Images by GIS and Deep Residual Learning. [in:] Iliadis L., Angelov P., Jayne C., Pimenidis E. (eds.), Proceedings of the 21st EANN (Engineering Applications of Neural Networks) 2020 Conference: EANN 2020, Proceedings of the International Neural Networks Society, vol. 2, Springer, Cham 2020, pp 274-291. https://doi.org/10.1007/978-3-030-48791-1_21.

[26] Zhao J., Fan W., Zhai X.: Identification of land-use characteristics using bicycle sharing data: A deep learning approach. Journal of Transport Geography, vol. 82, 2020, 102562. https://doi.org/10.1016/j.jtrangeo.2019.102562.

[27] Wu A.N., Biljecki F.: Roofpedia: Automatic mapping of green and solar roofs for an open roofscape registry and evaluation of urban sustainability. Landscape and Urban Planning, vol. 214, 2021, 104167. https://doi.org/10.1016/j.landurbplan.2021.104167. 
[28] Oyesiku O.O.: From Womb to Tomb. Olabisi Onabanjo University, Ago-Iwoye, Ogun State, Nigeria 2002.

[29] Campbell A., Both A., Sun Q.C.: Detecting and mapping traffic signs from Google Street View images using deep learning and GIS. Computers, Environment and Urban Systems, vol. 77, 2019, 101350. https://doi.org/10.1016/j.compenvurbsys.2019.101350.

[30] Mubin N.A., Nadarajoo E., Shafri H.Z.M., Hamedianfar A.: Young and mature oil palm tree detection and counting using convolutional neural network deep learning method. International Journal of Remote Sensing, vol. 40(19), 2019, pp. 7500-7515. https://doi.org/10.1080/01431161.2019.1569282.

[31] Li W., He C., Fang J., Zheng J., Fu H., Yu L.: Semantic Segmentation-Based Building Footprint Extraction Using Very High-Resolution Satellite Images and Multi-Source GIS Data. Remote Sensing, vol. 11(4), 2019, 403. https://doi.org/ 10.3390/rs11040403.

[32] Kearney S.P., Coops N.C., Sethi S., Stenhouse G.B.: Maintaining accurate, current, rural road network data: An extraction and updating routine using RapidEye, participatory GIS and DL. International Journal of Applied Earth Observation and Geoinformation, vol. 87, 2020, 102031. https://doi.org/10.1016/ j.jag.2019.102031.

[33] Servizi V., Petersen N.C., Pereira F.C., Nielsen O.A.: Stop detection for smartphone-based travel surveys using geo-spatial context and artificial neural networks. Transportation Research Part C: Emerging Technologies, vol. 121, 2020, 102834. https://doi.org/10.1016/j.trc.2020.102834.

[34] Malaainine M., Lechgar H., Rhinane H.: YOLOv2 Deep Learning Model and GIS Based Algorithms for Vehicle Tracking. Journal of Geographic Information System, vol. 13, 2021, pp. 395-409. https://doi.org/10.4236/jgis.2021.134022.

[35] Bi H., Shang W.-L., Chen Y., Wang K., Yu Q., Sui Y.: GIS aided sustainable urban road management with a unifying queueing and neural network model. Applied Energy, vol. 291, 2021, 116818. https://doi.org/10.1016/j.apenergy. 2021.116818.

[36] Yang Y., Rosenbaum M.S.: Artificial neural networks linked to GIS. [in:] Nikravesh M., Aminzadeh F., Zadeh L.A. (eds.), Soft Computing and Intelligent Data Analysis in Oil Exploration, Developments in Petroleum Science, vol. 51, Elsevier, 2003, pp. 633-650. https://doi.org/10.1016/S03767361(03)80032-8.

[37] Dixon B.: Applicability of neuro-fuzzy techniques in predicting ground-water vulnerability: a GIS-based sensitivity analysis. Journal of Hydrology, vol. 309(1-4), 2005, pp. 17-38. https://doi.org/10.1016/j.jhydrol.2004.11.010.

[38] Almasri M.N., Kaluarachchi J.J.: Modular neural networks to predict the nitrate distribution in ground water using the on-ground nitrogen loading and recharge data. Environmental Modelling \& Software, vol. 20(7), 2005, pp. 851-871. https://doi.org/10.1016/j.envsoft.2004.05.001. 
[39] Rohmat F.I.W., Labadie J.W., Gates T.K.: Deep learning for compute-efficient modeling of BMP impacts on stream-aquifer exchange and water law compliance in an irrigated river basin. Environmental Modelling \& Software, vol. 122, 2019, 104529. https://doi.org/10.1016/j.envsoft.2019.104529.

[40] Apaydin H., Sattari M.T.: Deep-learning GIS hybrid approach in precipitation modeling based on spatio-temporal variables in the coastal zone of Turkey. Climate Research, vol. 81, 2020, pp. 149-165. https://doi.org/10.3354/cr01612.

[41] Patault E., Landemaine V., Ledun J., Soulignac A., Fournier M., Ouvry J.-F., Cerdan O., Laignel B.: Integrating DL to GIS Modelling: An Efficient Approach to Predict Sediment Discharge at Karstic Springs under Different Land-Use Scenarios. EGU General Assembly, 2020. https://doi.org/10.5194/egusphereegu2020-467.

[42] Lei X., Chen W., Panahi M., Falah F., Rahmati O., Uuemaa E., Kalantari Z., Ferreira C., Rezaie F., Tiefenbacher J., Lee S., Bian H.: Urban flood modeling using deep-learning approaches in Seoul, South Korea. Journal of Hydrology, vol. 601, 2021, 126684. https://doi.org/10.1016/j.jhydrol.2021.126684.

[43] Stassopoulou A., Petrou M., Kittler J.: Bayesian and neural networks for geographic information processing. Pattern Recognition Letters, vol. 17(13), 1996, pp. 1325-1330. https://doi.org/10.1016/S0167-8655(96)00089-X.

[44] Choi J., Oh H.-J., Lee H.-J., Lee S.: Combining landslide susceptibility maps obtained from frequency ratio, logistic regression, and artificial neural network models using ASTER images and GIS. Engineering Geology, vol. 124, 2012, pp. 12-23. https://doi.org/10.1016/j.enggeo.2011.09.011.

[45] Hamdi Z., Brandmeier M., Straub Ch., Straub D., Berk M.: Coupling DL and GIS for forest damage assessment based on high-resolution remote sensing data. Geophysical Research Abstracts, vol. 21, 2019, p1-1.

[46] Bui D.T., Hoang N.-D., Martínez-Álvarez F., Ngo P.-T.T., Hoa P.V., Pham T.D., Samui P., Costache R.: A novel deep learning neural network approach for predicting flash flood susceptibility: A case study at a high frequency tropical storm area. Science of the Total Environment, vol. 701, 2020, 134413. https://doi. org/10.1016/j.scitotenv.2019.134413.

[47] Radke D., Hessler A., Ellsworth D.: FireCast: leveraging DL to predict wildfire spread. [in:] Proceedings of the Twenty-Eighth International Joint Conference on Artificial Intelligence (IJCAI-19), International Joint Conferences on Artificial Intelligence, 2019, pp. 4575-4581. https://doi.org/10.24963/ijcai.2019/636.

[48] Nhu V.-H., Hoang N.-D., Nguyen H., Ngo P.T.T., Bui T.T., Hoa P.V., Samui P., Bui D.T.: Effectiveness assessment of Keras based deep learning with different robust optimization algorithms for shallow landslide susceptibility mapping at tropical area. CATENA, vol. 188, 2020, 104458. https://doi.org/10.1016/j.catena. 2020.104458 . 
[49] Wu Z., Zhou Y., Wang H., Jiang Z.: Depth prediction of urban flood under different rainfall return periods based on deep learning and data warehouse. Science of the Total Environment, vol. 716, (2020), 137077. https://doi.org/10.1016/ j.scitotenv.2020.137077.

[50] Bragagnolo L., da Silva R.V., Grzybowski J.M.V.: Landslide susceptibility mapping with r.landslide: A free open-source GIS-integrated tool based on Artificial Neural Networks. Environmental Modelling \& Software, vol. 123, 2020, 104565. https://doi.org/10.1016/j.envsoft.2019.104565.

[51] Ma J., Cheng J.C.P.: Estimation of the building energy use intensity in the urban scale by integrating GIS and big data technology. Applied Energy, vol. 183, 2016, pp. 182-192. https://doi.org/10.1016/j.apenergy.2016.08.079.

[52] Re Cecconi F., Moretti N., Tagliabue L.C.: Application of artificial neutral network and geographic information system to evaluate retrofit potential in public school buildings. Renewable and Sustainable Energy Reviews, vol. 110, 2019, pp. 266-277. https://doi.org/10.1016/j.rser.2019.04.073.

[53] Kucklick J.-P., Müller J., Beverungen D., Mueller O.: Quantifying the impact of location data for real estate appraisal - a GIS-based deep learning approach. ECIS 2021 Research-in-Progress Papers, no. 23, 2021. https://aisel.aisnet.org/ ecis2021_rip/23.

[54] Zhang Y., Li S., Dong R., Deng H., Fu X., Wang Ch., Yu T., Jia T., Zhao J.: Quantifying physical and psychological perceptions of urban scenes using deep learning. Land Use Policy, vol. 111, 2021, 105762. https://doi.org/10.1016/ j.landusepol.2021.105762.

[55] Pepe M., Costantino D., Alfio V.S., Vozza G., Cartellino E.: A Novel Method Based on DL, GIS and Geomatics Software for Building a 3D City Model from VHR Satellite Stereo Imagery. ISPRS International Journal of Geo-Information, vol. 10(10), 2021, 697. https://doi.org/10.3390/ijgi10100697. 